\title{
Prevalência e aspectos epidemiológicos de depressão em idosos
}

\author{
Prevalence and epidemiological aspects of depression in the elderly \\ Prevalencia y aspectos epidemiológicos de la depresión en el anciano
}

\section{Resumo}

O estudo visou identificar a prevalência de depressão no ambulatório de Saúde do idoso, de um Centro de Especialidades Médicas (CEMEC), reconhecendo aspectos epidemiológicos de idosos com essa enfermidade. Tratouse de pesquisa do tipo observacional, transversal, descritivo e analítico em prontuários de pacientes atendidos no CEMEC, que faz parte do Sistema Único de Saúde. A amostra foi constituída de 155 idosos (idade igual ou superior a 60 anos, de ambos os sexos), com a coleta de dados por meio de um questionário, elaborado pelos pesquisadores, com as variáveis: número do prontuário, sexo, idade, estado civil, ocupação, religião e escolaridade. Foram analisados prontuários e observou-se que a prevalência de depressão na população estudada foi de $24,51 \%$. Com relação ao gênero, $18,71 \%$ eram mulheres e 5,81\% homens com diagnóstico de depressão. Os idosos de 60 a 69 anos são os mais acometidos, com 9,03\%, a maioria correspondia àqueles que se declaravam casados, $37,84 \%$, seguido dos viúvos, $29,73 \%$ e, quanto à ocupação, $82,86 \%$ dos pacientes com depressão são aposentados. Dessa forma, compreende-se que a equipe de saúde esteja atenta ao idoso quanto a problemas psiquiátricos para prevenir, diagnosticar e tratar adequadamente os pacientes com transtornos depressivos gerando maior qualidade de vida.

Palavras-chave: Atenção à saúde; Idoso; Depressão.

\section{Abstract}

The study aimed to identify the prevalence of depression in the Elderly Health outpatient clinic of a Medical Specialties Center (CEMEC), recognizing epidemiological aspects of elderly people with this disease. This was an observational, cross-sectional, descriptive and analytical research in the medical records of patients treated at CEMEC, which is part of the Unified Health System. genders), with the collection of data through a questionnaire, elaborated by the researchers, with the variables: medical record number, sex, age, marital status, occupation, religion and education. Medical records were analyzed and it was observed that the prevalence of depression in the studied population was $24.51 \%$. Regarding gender, $18.71 \%$ were women and $5.81 \%$ men diagnosed with depression. Elderly 
people aged 60 to 69 years are the most affected, with $9.03 \%$, the majority corresponded to those who declared themselves married, $37.84 \%$, followed by widowers, $29.73 \%$ and, regarding occupation, $82.86 \%$ of patients with depression are retired. Thus, it is understood that the health team is attentive to the elderly regarding psychiatric problems to prevent, diagnose and adequately treat patients with depressive disorders, generating a better quality of life.

Keywords: Delivery of health care; Aged; Depression.

\section{Resumen}

El estudio tuvo como objetivo identificar la prevalencia de depresión en el ambulatorio de Salud del Anciano de un Centro de Especialidades Médicas (CEMEC), reconociendo aspectos epidemiológicos de las personas mayores con esta enfermedad. Se trató de una investigación observacional, transversal, descriptiva y analítica en las historias clínicas de los pacientes atendidos en el CEMEC, que forma parte del Sistema Único de Salud (géneros), con la recolección de datos a través de un cuestionario, elaborado por los investigadores, con las variables: número de historia clínica, sexo, edad, estado civil, ocupación, religión y educación. Se analizaron las historias clínicas y se observó que la prevalencia de depresión en la población estudiada fue de $24,51 \%$. En cuanto al sexo, el 18,71\% eran mujeres y el 5,81\% hombres diagnosticados de depresión. Los ancianos de 60 a 69 años son los más afectados, con un $9,03 \%$, la mayoría correspondió a los que se declararon casados, el 37,84\%, seguidos de los viudos, el 29,73\% y, en cuanto a la ocupación, el $82,86 \%$ de los pacientes con depresión están jubilados. Así, se entiende que el equipo de salud está atento a las personas mayores en relación con los problemas psiquiátricos para prevenir, diagnosticar y tratar adecuadamente a los pacientes con trastornos depresivos, generando una mejor calidad de vida.

Palabras clave: Atención a la salud; Anciano; Depresión.

\section{Introdução}

O envelhecimento populacional é uma realidade em todo o mundo e, atualmente, esse fenômeno ocorre de maneira mais intensa nos países em desenvolvimento, como por exemplo, o Brasil (Ramos et al., 2019). Estima-se que em 2050 no mundo existirão cerca de 2,1 bilhões de pessoas com 60 anos ou mais, e em 2100 as projeções chegam aproximadamente a 3,2 bilhões de idosos (Souza Júnior et al., 2020).

Com esses dados, é importante salientar que longevidade não é necessariamente sinônimo de qualidade de vida, já que o aumento da idade pode se relacionar à maior incidência de doenças crônico-degenerativas, como a depressão (Galhardo et al., 2010).

A incidência de depressão maior é de aproximadamente $6 \%$ ao ano na população geral, sendo o risco de desenvolver um episódio depressivo é três vezes maior na recorrência em comparação ao primeiro evento (Malhi \& Mann, 2018). Dados do IBGE, em 2019, verificaram a prevalência do diagnóstico de depressão na população brasileira entre pessoas com 18 anos ou mais, sendo 14,7\% em mulheres e 5,9\% dos homens, na análise de acordo com a faixa etária a que teve maior prevalência foram os idosos entre 60 e 64 anos, 13,2\% do total de casos (IBGE, 2020).

O transtorno de humor depressivo deve ser encarado como um problema de saúde pública, afetando mais de 300 milhões de indivíduos no Mundo (OMS, 2020), sendo a prevalência estimada em idosos de cerca de 15\% para algum sintoma depressivo (Lima et al., 2016). De acordo com a Organização Mundial da Saúde (OMS) em 2016, que avaliou dados entre 2005 e 2015, a depressão já era considerada a principal causa específica de incapacidade social.

Especialmente na população geriátrica, a presença de depressão e sintomas de humor podem, além de gerar incapacidade social, acarretarem perda de autonomia e dependência funcional (Barbosa et al., 2013). Cerca de 20-30\% dos episódios depressivos apresentam um curso de evolução lenta e tais pacientes têm apresentado maiores consequências relacionadas à saúde, além de piores desfechos clínicos quando comparados aos quadros de depressão aguda (Schramm et al., 2020).

Em virtude do exposto, a pesquisa visou identificar a prevalência de depressão em idoosos no ambulatório de Saúde do idoso, do Centro de Especialidades Médicas do Centro Universitário do Estado do Pará (CEMEC), reconhecendo aspectos epidemiológicos de idosos com essa enfermidade acompanhadas neste ambulatório. 


\section{Metodologia}

Trata-se de um estudo do tipo observacional, transversal, descritivo e analítico em prontuários de pacientes atendidos no CEMEC, que faz parte do Sistema Único de Saúde (SUS).

A amostra foi constituída de 155 idosos, com a coleta de dados realizada após aceite institucional e aprovação do Comitê de Ética e Pesquisa do Centro Universitário do Estado do Pará (CAAE: 14136219.4.0000.5169).

Os pacientes elegíveis para este estudo foram aqueles cadastrados e atendidos no ambulatório de Saúde do Idoso do CEMEC e com idade igual ou superior a 60 anos, de ambos os sexos.

Foram analisados prontuários para revelar a prevalência de depressão entre estes pacientes. Após esta etapa, foram destacados apenas aqueles com o diagnóstico, segundo documentado. Excluíram-se pacientes sem diagnóstico de depressão.

Os dados foram coletados por meio de um questionário, elaborado pelos pesquisadores, com as seguintes variáveis: número do prontuário, sexo, idade, estado civil, ocupação, religião e escolaridade. Foram formuladas as seguintes questões, com respostas fechadas: "Paciente com diagnóstico de depressão? (Sim/Não)"; "Quanto pontuou na Escala de Depressão Geriátrica?".

Os pacientes foram pesquisados de acordo com os preceitos defendidos pela declaração de Helsinque e do Código de Nuremberg, considerando ainda as normas de pesquisa em seres humanos preceituadas pelo Conselho Nacional de Saúde (Resolução 466/2012).

Para comparação dos dados categóricos foram utilizados os testes não paramétricos Qui-quadrado (X2) e Teste G. Para análise estatística dos dados coletados, foram utilizados os softwares Excel@ 2010 e Word@ 2010, para análise dos dados e formatação dos gráficos e tabelas.

\section{Resultados}

Foram analisados 155 prontuários, do total de 1500 pacientes cadastrados no Ambulatório de Saúde do Idoso, o que corresponde à 10,33\%. Dentre esses, observou-se que a prevalência de depressão na população estudada foi de $24,51 \%$. Com relação ao gênero, 18,71\% eram mulheres e 5,81\% homens com diagnóstico de depressão (Gráfico1).

Gráfico 1: Distribuição quanto ao gênero dos pacientes atendidos no ambulatório de Saúde do Idoso.

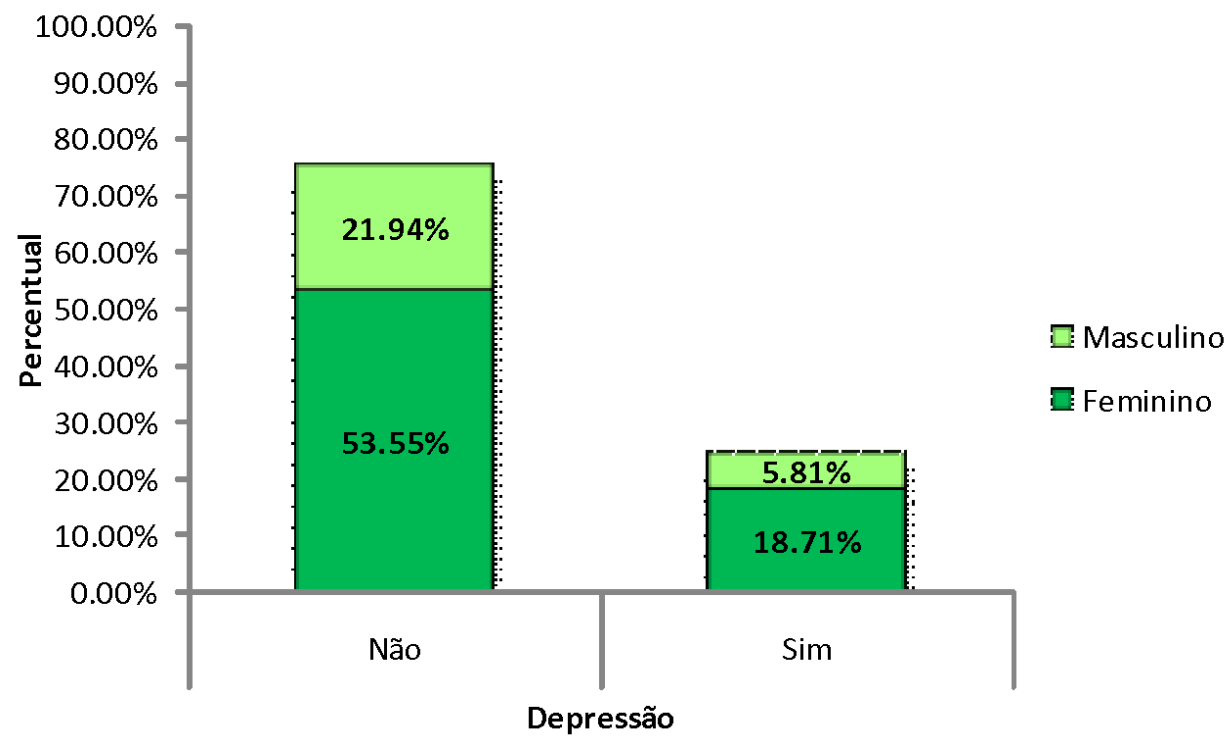

*p=0,961 (Qui-quadrado). Fonte: Protocolo de pesquisa. 
O Gráfico 2 mostram os pacientes que têm ou não depressão de acordo com sua faixa etária. Nota-se que entre os pacientes que não têm depressão os idosos com idade de 70 a 79 anos são os mais frequentes, com 31,61\%, já entre os pacientes que têm depressão, os idosos de 60 a 69 anos são os mais acometidos, com 9,03\%.

Gráfico 2: Distribuição quanto a faixa etária dos pacientes atendidos no ambulatório de Saúde do Idoso.

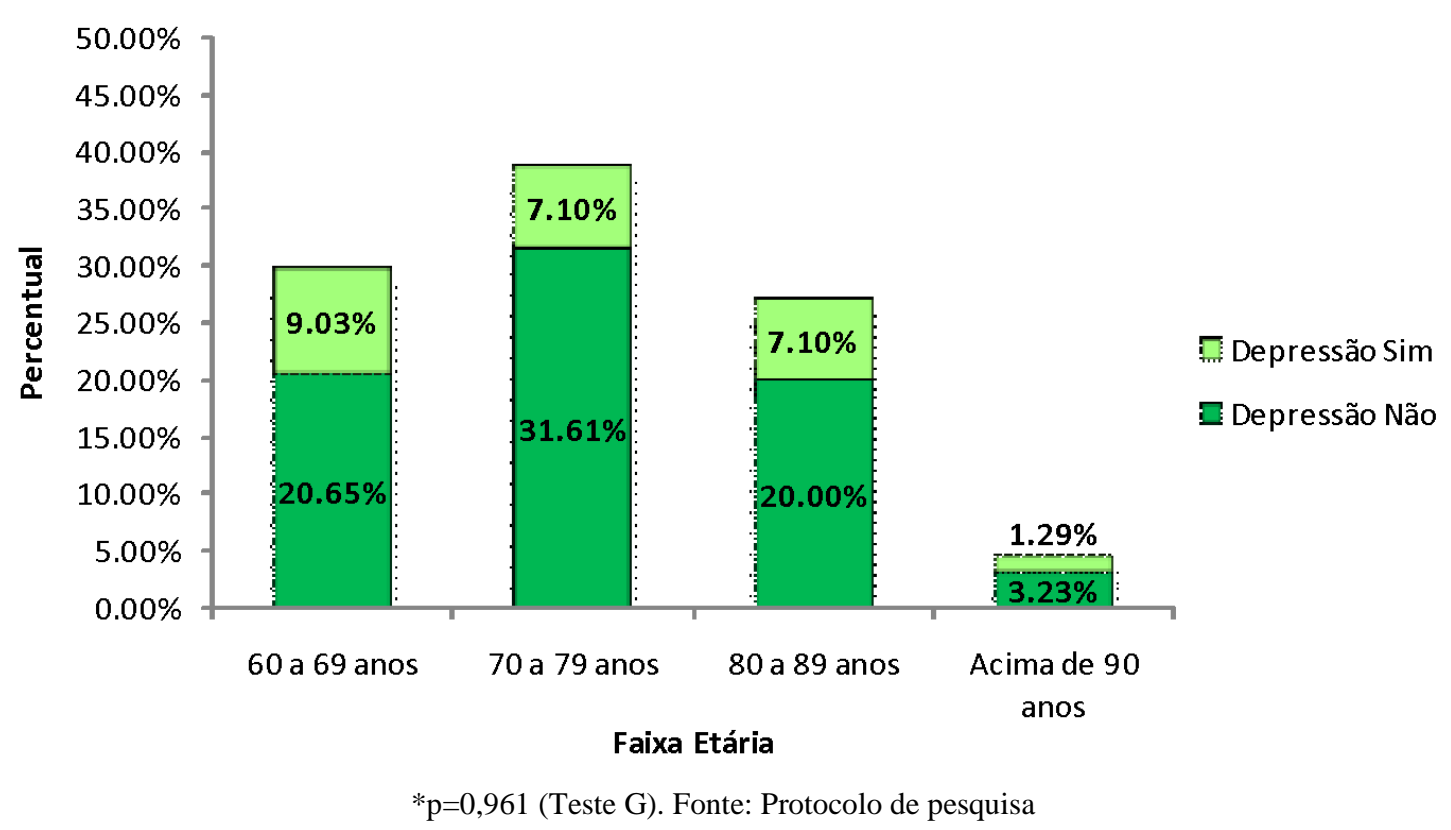

Quando avaliados a faixa etária, somente entre os pacientes com diagnóstico de depressão, os idosos com 60 a 69 anos são os mais frequentes, com $36,84 \%$, os quais são seguidos pelas faixas etárias de 70 a 79 e de 80 a 89 , onde ambas representam $28,95 \%$ dos pacientes depressivos. (Tabela 1 ).

Tabela 1: Distribuição quanto a faixa etária dos pacientes atendidos com diagnóstico de depressão.

\begin{tabular}{lcc}
\hline \multicolumn{1}{c}{ Faixa Etária } & Quantidade & Percentual \\
\hline 60 a 69 anos & 14 & $36,84 \%$ \\
70 a 79 anos & 11 & $28,95 \%$ \\
80 a 89 anos & 11 & $28,95 \%$ \\
Acima de 90 anos & 2 & $5,26 \%$ \\
\hline Total & $\mathbf{3 8}$ & $\mathbf{1 0 0 , 0 0 \%}$ \\
\hline
\end{tabular}

Fonte: Protocolo de pesquisa.

Dentre aqueles com depressão, 20,13\% foram classificados como tendo depressão leve, e 3,90\% apresentaram depressão severa (Gráfico 3). 
Gráfico 3: Proporção por escore na Geriatric Depression Scale (GDS) de pacientes atendidos no ambulatório de Saúde do Idoso.

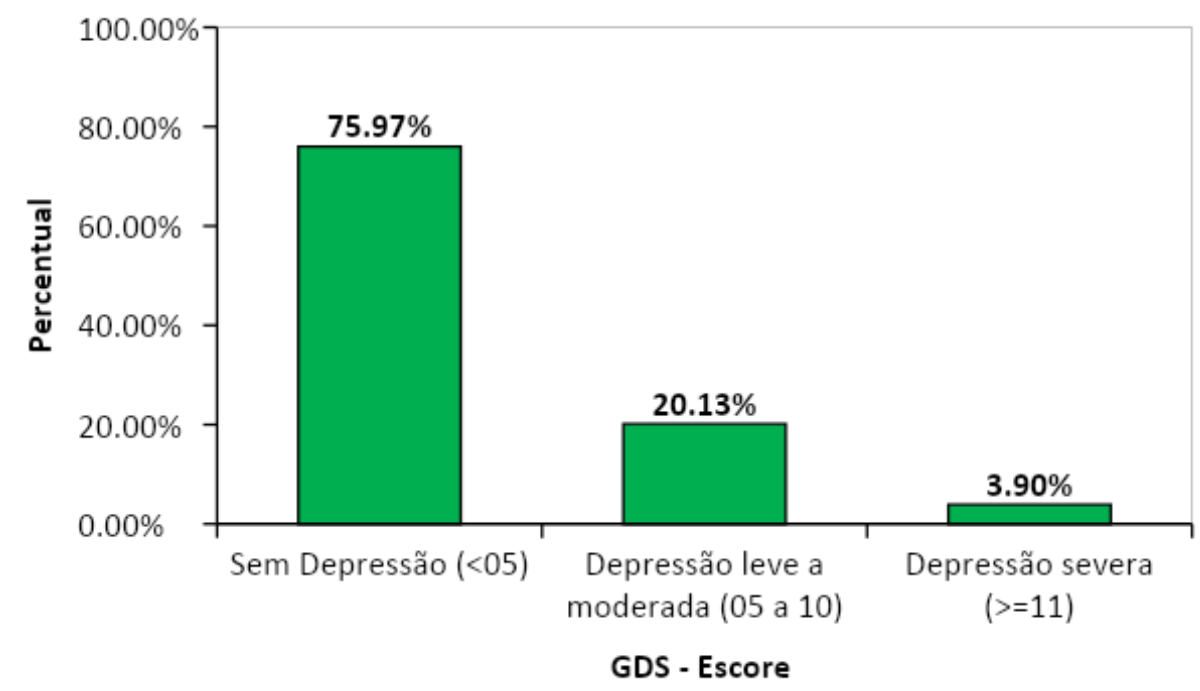

Nota: 1 (um) paciente não informou. Fonte: Protocolo de pesquisa.

Quando avaliado o estado civil, dos pacientes com depressão, notou-se que a maioria correspondia àqueles que se declaravam casados, 37,84\%, seguido dos pacientes viúvos, 29,73\% (Gráfico 4).

Gráfico 4: Distribuição quanto ao estado civil dos pacientes atendidos com diagnóstico de depressão.

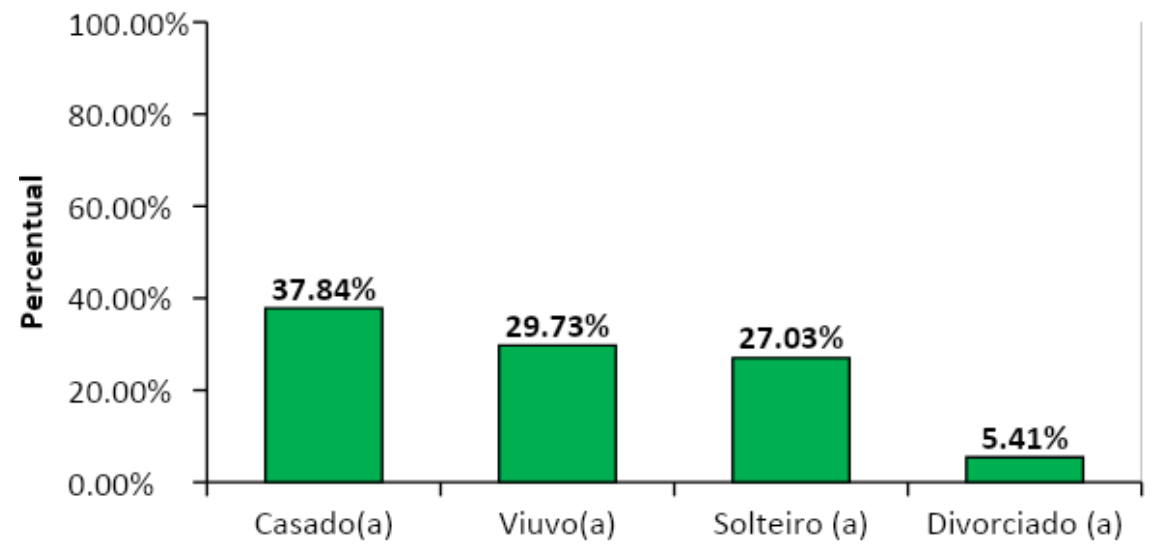

Estado Civil

Nota: 1 (um) paciente não informou. p<0,0001 (Teste G). Fonte: Protocolo de pesquisa.

Quanto à ocupação, 82,86\% dos pacientes com depressão são aposentados, os quais são seguidos por aqueles que se declaram como aposentados/ativos (vendedor) com 5,71\%, ou seja, já se aposentaram, mas continuam ativos no mercado de trabalho.

Três pacientes referiram estar ativamente no mercado de trabalho e não terem se aposentado, sendo respectivamente: um artesão, um autônomo e um vendedor (Tabela 2). 
Tabela 2: Distribuição quanto a ocupação dos pacientes atendidos com diagnóstico de depressão.

\begin{tabular}{lcc}
\hline \multicolumn{1}{c}{ Ocupação } & Quantidade & Percentual \\
\hline Aposentado & 29 & $82,86 \%$ \\
Aposentado/Ativo (Vendedor) & 2 & $5,71 \%$ \\
Ativo (Administrador) & 1 & $2,86 \%$ \\
Ativo (Artesão) & 1 & $2,86 \%$ \\
Ativo (Autônomo) & 1 & $2,86 \%$ \\
Ativo (Pescador) & 1 & $2,86 \%$ \\
\hline Total & $\mathbf{3 5}$ & $\mathbf{1 0 0 , 0 0 \%}$ \\
\hline
\end{tabular}

Nota: 3 (três) pacientes não informaram. p<0,0001 (Teste G). Fonte: Protocolo de pesquisa

\section{Discussão}

Inúmeras pesquisas mostram que a pessoa idosa é mais vulnerável a doenças psiquiátricas, em especial, a depressão. O declínio progressivo do humor caracteriza-se, biológica ou organicamente, por alterações fisiológicas em diversos sistemas desde o endócrino ao neurológico (Freitas et al., 2020).

Neste estudo, a prevalência de depressão na amostra foi de $24,51 \%$, porcentagem superior ao que a literatura aponta como 15\% de ocorrência na população mundial (Lima et al., 2016). Isso pode ter ocorrido por um viés de seleção, uma vez que a amostra foi selecionada especificamente de um ambulatório de geriatria de uma instituição de ensino, onde geralmente os pacientes atendidos são mais complexos e com multimorbidades.

Entretanto, a prevalência de depressão na pessoa idosa é bastante variável, podendo alcançar até $40 \%$ em algumas populações (Gonzalez et al., 2016). Essa variação na literatura pode ser decorrente do contexto social e cultural da população estudada, dos métodos diagnósticos, da utilização de diferentes escalas de medidas e do contexto em que a pesquisa foi realizada (Nóbrega et al., 2015).

Dentre os doentes foi observado que a maioria é do sexo feminino, 18,71\%, o que vai de acordo com dados do IBGE (2020), o qual afirma que este é um dado bastante conhecido na epidemiologia da depressão, realidade que também é confirmada em nível de literatura internacional (Sousa et al., 2010). Estudos ressaltam que a maior prevalência de depressão no sexo feminino, deve-se a fatores como: a maior longevidade destas, o maior tempo de exposição das mulheres a doenças crônicas, à viuvez e à solidão, o que facilita ao desencadeamento de sintomas depressivos (Güths et al., 2017). E, também, ao maior percentual de mulheres na terceira idade, a qual segundo a PNAD (2004) representa 56\% do contingente de idosos.

Estudos denotam que a típica "depressão feminina", é decorrente da exposição dessas idosas a fatores de risco (condição marital, baixa renda e escolaridade, condição de saúde, limitação funcional, baixo suporte social), e não somente a fatores biológicos, como privação de estrogênio (Oliveira et al., 2019).

Em relação a variável idade, a maior prevalência de depressão ocorre na faixa etária de 60-69anos, com 36,84\%, o que está de acordo com estudo de Laurindo et al. (2020) que encontrou a prevalência de depressão também maior nos idosos mais jovens, com 51\% dos casos entre 60 e 69 anos, $31 \%$ entre 70 e 79 anos e $18 \%$ acima de 80 anos.

O trabalho avaliou a presença de depressão, assim como o score na escala GDS e seu respectivo grau de severidade da depressão. A GDS-15 é uma versão curta da escala original e foi elaborada por Sheikh e Yesavage (1986), a partir dos itens que mais fortemente se correlacionam com o diagnóstico de depressão, possui sensibilidade de $81 \%$ e especificidade de $71 \%$, quando usado o ponto de corte 5/6, sendo um método com boa acurácia para rastreio e diagnóstico (Barbosa et al., 2013).

Devido ao fato de que idosos deprimidos são menos propensos a apresentarem sintomas afetivos e mais tendentes a 
mostrarem alterações cognitivas, perda de interesse ou, ainda, somatização quando comparados a adultos jovens (Fiske, Wetherell \& Gatz, 2009; Silva et al., 2021), sugere-se que as escalas e critérios diagnósticos são aplicáveis e úteis, porém não devem ser exclusivos, a anamnese e avaliação clínica detalhada, assim como a individualização do paciente são importantes na investigação dos sintomas de humor e não podem ser esquecidos.

Essa importância no diagnóstico de depressão conquista espaço em grandes estudos, principalmente, devido ao extremo de ideação suicida. Uma pesquisa sueca avaliou pacientes que tentaram suicídio e evidenciou que 52\% dos idosos preenchiam os critérios diagnósticos clássicos de depressão maior, na população de meia-idade e adultos jovens o diagnóstico foi obtido em 73\% e 76\%, respectivamente (Wiktorsson, 2021).

O estado civil mais encontrado entre os idosos pesquisados é a dos casados (37,84\%), seguidos por viúvos (29,73\%), solteiros $(27,03 \%)$ e divorciados $(5,41 \%)$. No entanto, quando somado o total de pacientes não casados, eles somam o total de $62,16 \%$, evidenciando maior prevalência. Essa realidade foi ratificada no estudo de Sousa et al. (2010) que encontrou uma ocorrência maior da patologia entre os não casados.

É dedutível que idosos casados devem ter menor risco para desenvolver depressão do que aqueles que não tinham cônjuges. Diversos estudos apontam que a presença de um companheiro, é visto como um fator protetor psicossocial, o que leva ao apoio mútuo entre os parceiros e o enfrentamento de situações adversas (Silva et al., 2017).

Em relação a ocupação, a maioria $(82,86 \%)$ era aposentada, realidade coexistente com outras pesquisas (Bretanha et al., 2015). A correlação entre aposentadoria e depressão já foi evidenciada em diversos estudos, publicação do Institute of Economics Affairs (IEA) em 2013, constatou que após a aposentadoria o risco de um indivíduo desenvolver depressão aumenta em cerca de $40 \%$, sendo o isolamento social e a falta de planejamento financeiro dois dos fatores que mais contribuem neste quadro.

De acordo com Bretanha et al. (2015), a aposentadoria é um fator protetor contra a depressão, pois a renda advinda é elemento fundamental para preservar a autonomia da população geriátrica. No entanto, os idosos, comumente, encontram-se menos favorecidos no que tange às finanças após a aposentadoria, o que resulta em declínio social. Além disso, vale ressaltar, que a inatividade econômica atinge diretamente a qualidade de vida dos idosos, já que sem renda estes se encontram impossibilitados de ter acesso a cuidados adequados e aquisição de medicamentos para o tratamento de doenças crônicas e incapacitantes (Bretanha et al., 2015).

\section{Conclusão}

Conclui-se que a prevalência de depressão foi de 24,51\%, 18,71\% eram mulheres e 5,81\% homens com diagnóstico de depressão. Os idosos de 60 a 69 anos são os mais acometidos, com 9,03\% e a maioria correspondia àqueles que se declaravam casados e aposentados.

É imprescindível que a equipe de saúde esteja atenta ao idoso quanto a problemas psiquiátricos, uma vez que a presença de doenças crônicas e o uso de múltiplos medicamentos são habituais na população geriátrica, tornando o diagnóstico e o próprio tratamento dos transtornos do humor mais complexos. Além disso a associação do impacto negativo na qualidade de vida e risco aumentado de suicídio mostram a importância do diagnóstico.

Desta maneira, prevenir, diagnosticar e tratar adequadamente os pacientes com transtornos depressivos geram maiores índices de qualidade de vida e autopercepção de qualidade em saúde dos pacientes. Por ser uma condição bastante prevalente nos pacientes idosos, as equipes de saúde que lidam com este público, em especial geriatras, devem estar capacitadas e disponíveis para realizar o rastreio destes sintomas, proporcionando assim melhor qualidade de vida a esta população. 


\section{Referências}

Barbosa, F. B. M., Biermann, L. S., Peixoto Júnior, A. A., Almeida, G. H. (2013). Depressive disorer in elderly people: screening, diagnosis and epidemiological aspects. Geriatr Gerontol Aging, 7:228-233.

Bretanha, A. F., Facchini, L. A., Nunes, B. P., Munhoz, T. N., Tomasi, E., \& Thumé, E. (2015). Sintomas depressivos em idosos residentes em áreas de abrangência das Unidades Básicas de Saúde da zona urbana de Bagé, RS. Revista Brasileira de Epidemiologia, $18,1-12$.

Fiske, A., Wetherell, J. L., \& Gatz, M. (2009). Depression in older adults. Annual review of clinical psychology, 5, 363-389.

Freitas, C. B. de, Veloso, T. C. P., da Silva Segundo, L. P., de Sousa, F. P. G., Galvão, B. S., \& Nagaishi, C. Y. (2020). Prevalence of depression between institutionalized elderly. Research, Society and Development, 9(4), 8.

Galhardo, V. A. C., Mariosa, M. A. S., \& Takata, J. P. I. (2010). Depressão e perfil sociodemográfico e clínico de idosos institucionalizados sem déficit cognitivo. Rev Med Minas Gerais, 20(1), 16-21.

González, A. C. T., Ignácio, Z. M., Jornada, L. K., Réus, G. Z., Abelaira, H. M., Santos, M. A. B. D., \& Quevedo, J. L. D. (2016). Depressive disorders and comorbidities among the elderly: a population-based study. Revista Brasileira de Geriatria e Gerontologia, 19, 95-103.

Güths, J. F. D. S., Jacob, M. H. V. M., Santos, A. M. P. V. D., Arossi, G. A., \& Béria, J. U. (2017). Perfil sociodemográfico, aspectos familiares, percepção de saúde, capacidade funcional e depressão em idosos institucionalizados no Litoral Norte do Rio Grande do Sul, Brasil. Revista Brasileira de Geriatria e Gerontologia, 20, 175-185.

IBGE. (2020). Pesquisa nacional de saúde: 2019: percepção do estado de saúde, estilos de vida, doenças crônicas e saúde bucal: Brasil e grandes regiões / IBGE, Coordenação de Trabalho e Rendimento. 69.

IBGE. (2004). Pesquisa Nacional por Amostras de Domicílios 2004. http://www.ibge.gov.br.

Laurindo, M. V., Lopes, R. E., \& Rocha, R. R. (2020). Perfil Epidemiológico de Idosos com Transtornos Depressivos em um Município do Nordeste Brasileiro. Brazilian Journal of Development, 6(1), 2945-2961.

Lima, A. M. P., Ramos, J. L. S., Bezerra, I. M. P., Rocha, R. P. B., Batista, H. M. T., \& Pinheiro, W. R. (2016). Depressão em idosos: uma revisão sistemática da literatura. Revista de Epidemiologia e Controle de Infecção, 6(2), 97-103.

Malhi, G. S. \& Mann, J. J. (2018). Depression. Lancet. 24, 392(10161):2299-2312. 10.1016/S0140-6736(18)31948-2.

Nóbrega, I. R. A. P. D., Leal, M. C. C., Marques, A. P. D. O., \& Vieira, J. D. C. M. (2015). Fatores associados à depressão em idosos institucionalizados: revisão integrativa. Saúde em Debate, 39, 536-550.

Oliveira, D. V. D., Pivetta, N. R. S., Oliveira, G. V. D. N. D., Silva, D. A. D., Nascimento Júnior, J. R. A. D., \& Cavaglieri, C. R. (2019). Fatores intervenientes nos indicativos de depressão em idosos usuários das unidades básicas de saúde de Maringá, Paraná, 2017. Epidemiologia e Serviços de Saúde, $28, \mathrm{e} 2018043$.

OMS. (2020). https://www.paho.org/pt/topicos/depressao

Ramos, F. P., da Silva, S. C., de Freitas, D. F., Gangussu, L. M. B., Bicalho, A. H., de Oliveira Sousa, B. V., ... \& Guimarães, T. A. (2019). Fatores associados à depressão em idoso. Revista Eletrônica Acervo Saúde, (19), e239-e239.

Schramm, E., Klein, D. N., Elsaesser, M., Furukawa, T. A., \& Domschke, K. (2020). Review of dysthymia and persistent depressive disorder: history, correlates, and clinical implications. The Lancet Psychiatry, 7(9), 801-812.

Silva, A. R., Sgnaolin, V., Nogueira, E. L., Loureiro, F., Engroff, P., \& Gomes, I. (2017). Doenças crônicas não transmissíveis e fatores sociodemográficos associados a sintomas de depressão em idosos. Jornal brasileiro de Psiquiatria, 66, 45-51.

Silva, B. C. M. da, Anjos, I. C. S. dos, Neto, G. D. S. P., Santana, D. S., de Souza Araújo, J., da Silva Alves, D. J., \& de Aguiar, V. F. F. (2021). Importância da identificação do diagnóstico de enfermagem ao paciente com depressão senil na atenção básica. Research, Society and Development, 10(2), e53510212770e53510212770.

Sousa, M., Nunes, A., Guimarães, A. I., Cabrita, J. M., Cavadas, L. F., \& Alves, N. F. (2010). Depressão em idosos: prevalência e factores associados. Revista Portuguesa de Medicina Geral e Familiar, 26(4), 384-91.

Souza Júnior, E. V., Cruz, D. P., Siqueira, L. R., Rosa, R. S., Silva, C. D. S., Sawada, N. O. (2021). Association between common mental disorders and quality of life in older adults. Rev Esc Enferm USP. 13, 55, e20210057. 10.1590/1980-220X-REEUSP-2021-0057.

Wiktorsson, S., Strömsten, L., Renberg, E. S., Runeson, B., \& Waern, M. (2021). Clinical characteristics in older, middle-aged and young adults who present with suicide attempts at psychiatric emergency departments: a multisite study. The American Journal of Geriatric Psychiatry, 9:S1064-7481(21)00419-X. 10.1016/j.jagp.2021.08.001. 